\title{
Operationalizing an Implementation Framework to Disseminate a Care Coordination Program for Rural Veterans
}

\author{
Chelsea Leonard, $P h D^{7}$, Heather Gilmartin, $P h D, N P^{7}$, Marina McCreight, MPH' , Lynette Kelley, NP', \\ Brandi Lippmann, $\mathrm{MA}^{7}$, Ashlea Mayberry, $\mathrm{RN}{ }^{7}$, Andrew Coy, BS ${ }^{7}$, Emily Lawrence, $M P H^{7}$, and \\ Robert E. Burke, MD, $M S^{2,3}$
}

'Denver/Seattle Center of Innovation for Veteran-Centered and Value Driven Care, VA Eastern Colorado Healthcare System, Denver, CO, USA; ${ }^{2}$ Center for Health Equity Research and Promotion (CHERP), Corporal Crescenz VA Medical Center, Philadelphia, PA, USA; ${ }^{3}$ Hospital Medicine Section, Division of General Internal Medicine, University of Pennsylvania Perelman School of Medicine, Philadelphia, USA.

\begin{abstract}
OBJECTIVE: Understanding how to successfully implement care coordination programs across diverse settings is critical for disseminating best practices. We describe how we operationalized the Practical Robust Implementation and Sustainability Model (PRISM) to guide the assessment of local context prior to implementation of the rural Transitions Nurse Program (TNP) at five facilities across the Veterans Health Administration (VHA).
\end{abstract}

METHODS: We operationalized PRISM to create qualitative data collection techniques (interview guides, semistructured observations, and a group brainwriting premortem) to assess local context, the current state of care coordination, and perceptions of TNP prior to implementation at five facilities. We analyzed data using deductive-inductive framework analysis to identify themes related to PRISM. We adapted implementation strategies at each site using these findings.

RESULTS: We identified actionable themes within PRISM domains to address during implementation. The most commonly occurring PRISM domains were "organizational characteristics" and "implementation and sustainability infrastructure." Themes included a disconnect between primary care and hospital inpatient teams, concerns about work duplication, and concerns that one nurse could not meet the demand for the program. These themes informed TNP implementation.

CONCLUSIONS: The use of PRISM for preimplementation site assessments yielded important findings that guided adaptations to our implementation approach. Further, barriers and facilitators to TNP implementation may be common to other care coordination interventions. Generating a common language of barriers and facilitators in care coordination initiatives will enhance generalizability and establish best practices.

IMPACT STATEMENTS: TNP is a national intensive care coordination program targeting rural Veterans. We operationalized PRISM to guide implementation efforts. We effectively elucidated facilitators, barriers, and unique contextual factors at diverse VHA facilities. The use of PRISM enhances the generalizability of findings across care settings and may optimize implementation of care coordination interventions in the VHA.

Published online May 16, 2019
KEY WORDS: care coordination; care transitions; qualitative research; implementation science; rural Veterans.

J Gen Intern Med 34(Suppl 1):S58-S66

DOI: $10.1007 / \mathrm{s} 11606-019-04964-1$

(c) Society of General Internal Medicine (This is a U.S. government work and not under copyright protection in the U.S.; foreign copyright protection may apply) 2019

\section{INTRODUCTION}

The transition from hospital to home is a high-risk period for patients. ${ }^{1,2}$ Challenges to a safe transition include lack of communication regarding self-care needs, poor understanding of home care strategies, and difficulties adjusting to new medications. ${ }^{3,4}$ Many of these challenges can be addressed through care coordination, or the deliberate organization of a patient's care activities among providers. ${ }^{5}$ Care coordination enhances quality of care, reduces costs, and improves patient quality of life. ${ }^{6}$ Several care coordination interventions have successfully reduced hospital readmissions, ${ }^{7-9}$ but very few have been disseminated to diverse settings. ${ }^{3,10}$ Improving care coordination is a priority in the Veterans Health Administration (VHA), but there is little consensus on how to best disseminate care coordination interventions across large health care systems. ${ }^{7,11,12}$

Adapting interventions to local contexts improves program implementation. ${ }^{13-15}$ Implementation science tools and frameworks provide a standardized structure for understanding how contextual differences at diverse sites may affect implementation of an intervention. ${ }^{16,17}$ Created by national experts and informed by prior implementation science work, the Practical Robust Implementation and Sustainability Model (PRISM) ${ }^{15}$ provides a framework for applying implementation science principles. PRISM describes six key domains to evaluate in disseminating evidence-based interventions: implementation and sustainability infrastructure of the receiving organization, organizational perspective of the intervention, patient perspective of the intervention, organizational characteristics, external environment, and patient characteristics. ${ }^{15}$ Although PRISM has shown promise as a useful framework for exploring barriers and facilitators in 
case studies, ${ }^{18,19}$ questions remain about how to successfully operationalize the framework in multisite studies.

This project sought to address these two gaps: the lack of published data on experiences using rigorous implementation science methods to inform dissemination of promising care coordination interventions, and how to operationalize PRISM for this purpose. PRISM guided the implementation of the rural Transitions Nurse Program (TNP) across five diverse VHA facilities. TNP is a multi-component, nurse-led intensive care coordination intervention designed to improve care transitions for rural Veterans who are hospitalized at VHA hospitals and subsequently discharged to their rural residence and care setting. ${ }^{20,21}$ The transition from hospital to home is especially challenging for rural patients, who may experience medication gaps due to limited availabilities of medication in rural areas, inadequate discharge plans, lack of communication between hospital and primary care providers, and difficulty obtaining follow-up care. ${ }^{20}$ Like most successful care coordination programs, TNP addresses multiple barriers to an ideal transition of care and encompasses both pre- and post-discharge components. ${ }^{4}$ The TNP intervention consists of four core components carried out by a Transitions Nurse (TN) based at an urban VA medical center. These include meeting with the Veteran in the hospital to assess and address readmission risks, partnering with inpatient medical teams to identify post-discharge needs and coordinate a follow-up with a VA primary care provider, engaging with the rural Patient Aligned Care (PACT) team by sending hospital records and calling to discuss patient needs, and a follow-up phone call with the Veteran to revisit goals set during hospitalization and assess current symptoms. In this paper, we discuss how we operationalized PRISM to identify barriers, facilitators, and important contextual factors prior to TNP implementation. We then describe how we tailored our implementation efforts to address the most common barriers, facilitators, and contextual factors at each site. Our findings may apply to dissemination of care coordination interventions in a variety of settings.

\section{METHODS}

\section{Pre-implementation Assessment Design}

We operationalized PRISM by creating definitions for each of the six PRISM domains. We identified data categories and sources necessary to assess each domain. We collected and analyzed pre-implementation data with the goal of learning about contextual factors at each site.

We applied a rapid ethnographic approach to assess context using a variety of methods. ${ }^{22}$ We designed key informant (KI) interviews to elicit information on organizational perspective of the intervention, organizational characteristics, implementation and sustainability infrastructure, external environment, and barriers and facilitators to TNP implementation and success (Appendix, Key
Informant Interview Guide). We pilot tested the KI interview guide among colleagues to ensure that questions were easily understood. KI interviews were conducted over the phone with participants at both VA hospitals and PACT clinics, recorded, and transcribed verbatim. We designed observations of discharge processes and follow-up appointments to assess patient characteristics, implementation and sustainability infrastructure, and organizational characteristics. We piloted the observation process locally prior to site visits. Observers took detailed notes on process and context of discharge planning, including the types and nature of interactions observed. Observations were conducted at both VA hospitals and PACT clinics. Veteran interviews were intended to gather information on patient perspective of the intervention and patient characteristics. ${ }^{21}$ Finally, the brainwriting premortem activity was designed to identify barriers to program success. Brainwriting is a silent, written group brainstorming activity. The brainwriting premortem was developed and piloted in Denver to identify reasons TNP might fail. Each brainwriting session was followed by a discussion, which was recorded, and transcribed verbatim. $^{23}$

\section{Data Analysis}

We used inductive-deductive framework analysis to identify themes across KI interviews, observation notes, Veteran interviews, brainwriting papers, and transcripts from postbrainwriting discussions. Deductive codes were based on the six PRISM domains and factors that may be encapsulated within those domains (15; Table 3), barriers, and facilitators. Qualitative analysts created emergent codes to label additional contextual factors, barriers, and facilitators. Intercoder consensus was built through team discussion by resolving points of disagreement after analysts independently coded the same 11 documents, representing $12 \%$ of all qualitative documents. ${ }^{24}$ Atlas.ti V 7.5.18 ${ }^{25}$ was used to manage qualitative data.

\section{Identification of Adaptations Using Qualitative Data}

Key contextual factors, facilitators, and barriers were compared across sites. We shared these data with sites at an inperson training session in Denver 1 month after the completion of data collection. Data were used to brainstorm actionable adaptations to the implementation process. TN's from all sites continued to discuss potential adaptations on weekly phone calls with facilitators from the Denver TNP team. TNP received quality improvement status from the VA Office of Rural Health (ORH), which funded this project. All findings were used for operations purposes. Participants were informed that their participation was voluntary and assured that their contributions would remain confidential. 


\section{RESULTS}

Our efforts to operationalize PRISM resulted in working definitions of each of the six domains. Table 1 shows the data categories and types of data collected to assess each domain. Table 2 shows the number of each qualitative data type collected. We identified themes related to five of the six PRISM domains using data from all sources. We were unable to identify themes related to "patient perspective of the intervention" due to a limited number of interviews with Veterans. PRISM themes highlight potential barriers and facilitators to TNP implementation and identify areas in which TNP can improve transitional care for rural Veterans. Quotes to illustrative each theme are provided in Table 3.

\section{Themes Related to Organizational Characteristics}

\begin{abstract}
Disconnect Between Primary Care Teams and Hospital Inpatient Teams. Hospital-based participants noted a lack of knowledge of workload, resource availability, and processes in primary care. They did not know what follow-up capabilities were in place when recommending future care for hospitalized patients. Participants in primary care settings described a disconnect between hospital expectations for discharge follow-up and the reality of what they could provide. They described confusion around who to contact for additional information, or difficulties and frustrations when contacting inpatient hospital teams (quote A1, Table 3).
\end{abstract}

\section{Areas for Improvement in Current Transition of Care} Processes. Given the disconnect between hospital and primary care settings, participants at all sites described areas for improvement in transitional care processes. One site stressed the need for better medication education since pharmacy was short-staffed. The remaining sites emphasized poor coordination of discharge supplies and follow-up services, and communication barriers within and between ser- vices. Poor coordination was reported to result in challenges for patients trying to obtain medication, supplies, or follow-up care (quote A2, Table 3).

\section{Themes Related to Organizational Perspective of the Intervention}

Positive to Mixed Reaction to the Program. Many participants expressed strong enthusiasm for the program. They felt that TNP addressed a clear gap in transitional care for rural Veterans and thought that TNP had the potential to improve communication between hospitals and PACT sites. Some participants stated that positive program outcomes would depend on how TNP was rolled out, what kind of support it received, and who was selected for the TN position. In particular, participants felt success hinged upon the ability of the TN to provide clear education about the program, patient eligibility, and how the TN could augment existing work processes (quote B1, Table 3).

Concerns About Work Duplication. One of the most common concerns about the program was that it would duplicate work, overlapping with existing programs. For example, participants at rural clinics worried that the TN follow-up call would duplicate the PACT nurses' post-discharge call. Hospital staff worried that the TN's discharge planning duties would overlap with the responsibilities of other roles involved in discharge (quote B2, Table 3).

Concerns That One Nurse Could Not Meet the Demand for the Program. Participants at most sites emphasized that there were many more high-risk rural patients than one nurse could enroll in the TNP. Thus, they worried that the TN would be overwhelmed and overburdened. Some participants stated the necessity for the TN to say "no" when necessary, and others suggested that the TN clearly delineate eligibility criteria (quote B3).

Table 1 PRISM Working Definitions and Types of Data Collected

\begin{tabular}{|c|c|c|}
\hline PRISM domain & TNP working definition & Data types \\
\hline $\begin{array}{l}\text { Organizational perspective } \\
\text { of the intervention }\end{array}$ & $\begin{array}{l}\text { Staff's perspectives on the evidence base for the } \\
\text { intervention, potential barriers to frontline staff } \\
\text { and the usefulness of the intervention to the local } \\
\text { context }\end{array}$ & $\begin{array}{l}\text { Key informant } \\
\text { interviews, brainwriting }\end{array}$ \\
\hline $\begin{array}{l}\text { Patient perspective of the } \\
\text { intervention }\end{array}$ & $\begin{array}{l}\text { Patient thoughts on key intervention components, } \\
\text { and initial reactions to the program }\end{array}$ & Veteran interviews \\
\hline Organizational characteristics & $\begin{array}{l}\text { The dynamic between teams, financial and structural } \\
\text { health of the organization, organizational culture and } \\
\text { the history of trying or resisting new programs }\end{array}$ & $\begin{array}{l}\text { Observations of hospital and } \\
\text { PACT teams working, key } \\
\text { informant interviews, brainwriting }\end{array}$ \\
\hline Patient characteristics & $\begin{array}{l}\text { Patient demographics, competing programs and } \\
\text { demands on the patients, and stories of care transitions } \\
\text { for patients that may be affected by the TNP }\end{array}$ & $\begin{array}{l}\text { Veteran interviews, key informant } \\
\text { interviews, brainwriting }\end{array}$ \\
\hline External environment & $\begin{array}{l}\text { Current regulatory environment, community services/ } \\
\text { demographics may impact the organizational culture } \\
\text { and/or implementation and sustainability of an intervention }\end{array}$ & $\begin{array}{l}\text { Observations in hospital and PACT } \\
\text { sites, key informant interviews, } \\
\text { brainwriting }\end{array}$ \\
\hline $\begin{array}{l}\text { Implementation and } \\
\text { sustainability infrastructure }\end{array}$ & $\begin{array}{l}\text { Relationship and communication between individuals } \\
\text { in the infrastructure, implementation infrastructure, and } \\
\text { routine performance measurement and data sharing }\end{array}$ & $\begin{array}{l}\text { Observations in hospital and PACT } \\
\text { sites, key informant interviews, } \\
\text { group brainwriting }\end{array}$ \\
\hline
\end{tabular}


Table 2 Number of Each Qualitative Data Type Collected at Each Site

\begin{tabular}{|c|c|c|c|c|c|c|c|}
\hline & & Total $(n)$ & Site A (n) & Site B (n) & Site C (n) & Site D (n) & Site E (n) \\
\hline \multirow[t]{2}{*}{ Key informant interview } & Hospital & 21 & 3 & 4 & 3 & 5 & 6 \\
\hline & PACT & 20 & 4 & 2 & 4 & 6 & 4 \\
\hline \multirow[t]{2}{*}{ Observations } & Hospital & 21 & 4 & 4 & 4 & 4 & 5 \\
\hline & PACT & 11 & 3 & & 2 & 4 & 2 \\
\hline \multirow{3}{*}{$\begin{array}{l}\text { Brainwriting sessions (session includes papers and } \\
\text { transcript) } \\
\text { Veteran interviews }\end{array}$} & Hospital & 6 & 2 & 1 & 1 & 1 & 1 \\
\hline & PACT & 7 & 2 & 1 & 1 & 1 & 2 \\
\hline & & 7 & 1 & 1 & 1 & 2 & 2 \\
\hline
\end{tabular}

\section{Themes Related to Implementation and Sustainability Infrastructure}

Weak Infrastructural Support for Coordination of Care Between Hospitals and PACT Sites. Participants at most sites described communication difficulties between VA tertiary and primary care sites. In some cases, medical records were not shared because the hospital and primary care site were in different VA regions. This made it difficult for inpatient hospital teams to share information, and for participants in primary care to understand the details of hospitalizations. In other cases, there was a lack of familiarity with the needs of hospital or primary care clinicians and a lack of knowledge of who to contact with questions. This caused frustration when additional information about a patient was needed. This theme is related to the theme disconnect between primary care teams and hospital inpatient teams (quote $\mathrm{C} 1$, Table 3).

Concerns About Infrastructural Support for the Program. Sites were concerned about infrastructural support, including workspace, resources, and program sustainment after grant funding for the transition nurse position ended. Participants suggested that the Transitions Nurse build relationships with colleagues with strong institutional knowledge of care transition processes. Some participants described strong leadership interest in TNP and long-term plans to expand the TN position, while at other sites, participants described a need for positive program outcomes for continued program support (quote C2, Table 3).

\section{Themes Related to Patient Characteristics}

Difficulty Contacting Patients. All sites noted difficulty reaching rural Veterans as they often did not answer their phone, had poor telephone reception, or were hard of hearing. Some participants stated that Veteran contact information is often outdated in hospital records, or that patients sometimes return home with friends or family with different telephone numbers. Others suggested that some Veterans do not want to be contacted and might be unresponsive (quote D1, Table 3).
Transportation Challenges. Most sites also described a lack of reliable transportation among rural Veterans. Participants felt that low socioeconomic status of a large portion of the rural Veteran population was an important contributor to transportation issues. Participants noted that some rural Veterans live far from their VHA primary care facility and choose not to attend follow-up appointments (quote D2, Table 3).

\section{Themes Related to External Environment}

Impact of the Veteran's Choice Program. Participants at most sites discussed the potential impact of the Veteran's Choice Program, which allows Veterans to seek care outside of the VA. This program has increased non-VA healthcare utilization by Veterans. They described the need for a program like TNP to coordinate care between VA and community settings and wondered if TNP could fill this gap. They noted that many Veterans utilize community resources, but it was difficult to know which community services the Veteran qualified for or received (quote E1, Table 3 ).

Difficulties Recruiting and Retaining Providers in Rural Areas. Difficulties recruiting and retaining providers in the VA in rural settings were an important structural barrier, creating large patient panels for existing providers and limiting the availability of follow-up appointments. This was sometimes so impactful that the VA had to contract with outside providers to provide primary care for rural Veterans. Participants at contracted clinics expressed concern that TNP would increase their work load and necessitate contract renegotiation (quote E2).

\section{DISCUSSION}

Using PRISM to evaluate site context yielded important insights of potential barriers and facilitators to implementation of a care coordination program and helped identify crucially important adaptations. We could anticipate potential challenges, such as perceived work duplication, concerns that one nurse could not meet demand for the program, weak infrastructural support for care coordination, and difficulties related to patient characteristics. 
Table 3 Illustrative Quotes for Themes Related to PRISM Domains

\begin{tabular}{ll}
\hline \hline PRISM domain & Theme \\
\hline Organizational characteristics & $\begin{array}{l}\text { Disconnect between primary care teams and } \\
\text { hospital inpatient teams }\end{array}$
\end{tabular}

Organizational characteristics

for improvement in current transition of care processes

Organizational perspective of the intervention

Organizational perspective of the intervention

Organizational perspective of the intervention Implementation and sustainability infrastructure

Implementation and sustainability infrastructure

Patient characteristics

Difficulty contacting patients demand for the program Weak infrastructural support for coordination of care between hospitals and PACT sites

Concerns about infrastructural support for the program

Patient characteristics

Transportation challenges

External environment

Impact of the Veteran's Choice Program

Difficulties recruiting and retaining providers in rural areas

\section{Quote}

I think there's a disconnect with the understanding of how the movements of how [PACT clinics] work. Because we feel like we're on an island so we require a lot of non-VA agencies to help out and fill the gaps, where at the main center, they have more access to those services through the VA.

-PACT anonymous (brainwriting premortem)

A lot of times, what we have happen at discharge as well is they are not given the supplies that they need necessarily, so we do our twoday call and they're like you know, I don't have this, I don't have my oxygen supplies, I need this, I need this and those are things that could have been initiated from the inpatient side...

-PACT Nurse Manager (key informant interview)

Well, I think it would make the patients more likely to get the right care, I mean there's definitely a higher chance of them having the right follow-up and getting back down there if somebody's watching them and of course, I want to be honest, primary care really needs all the help it can get just to keep it, their nose above water, it's tough. -PACT Nurse Practitioner (key informant interview)

I think the role is awesome and much needed, but we have PACT care managers, and this is some of what they're supposed to be doing, so what's that all about?

- Hospital anonymous (brainwriting premortem)

Large volume of Veterans to manage.

- Hospital anonymous (brainwriting premortem)

One issues I would wish I could do, but we've struggled with this, is actually scheduling the follow-up appointment before the patient is discharged, and if we could do that, I would rate it higher than 8 , but there's too many factors that got in the way of us being able to do that...

- Hospital social worker (key informant interview)

I think that the main barrier for us to hire additional staff is always space, so we have a tight sort of area here and I would say that space is at a premium, but I think that the value of this position would encourage our facility to find a space for this person...

- Hospital clinician (key informant interview)

Sometimes, working phone number that's good, we send letters, but I would probably venture to say about $20,25 \%$ of our veterans are illiterate. It makes educating and following up very difficult when you have that type of combination that you often see with the rural veterans...

-PACT Nurse Manager (key informant interview)

What was brought up with transportation to appointments and that's more, you know, it's supposed to be rural based and that's your whole intent, we have a lot of transportation issues and we're probably not alone...

-PACT Nurse Manager (key informant interview)

The other problem is sometimes also if we have a patient through Choice go to an outside facility for services, we don't always know when that patient is getting discharged either until the day of or the day after and that's kind of difficult, too

- Hospital clinician (key informant interview)

I think, I don't know if it's so much retention, you know, they've been able to create some more provider positions. I think it's, sometimes it's hard to recruit, and sometimes it is hard to retain them in some of our rural areas...

- Hospital Registered Nurse (key informant interview)
At sites concerned with role duplication, we helped TN's create clear role descriptions and brainstorm ways to utilize existing infrastructure. At sites concerned that the TN would be overwhelmed with the number of eligible patients, we helped the TN's modify program enrollment criteria. Modifications to enrollment criteria were the most prevalent adaptation in the implementation process. At sites concerned with poor communication structure between hospitals and PACT sites, we recommended that TN's provide informational sessions at PACT sites to engage stakeholders and initiate relationships. This adaptation required more time and resources than others, but TN's visited at least some PACT clinics. Making these adaptations early in the implementation process helped to roll out TNP quickly at each site. Some TN's met enrollment goals within 2 months of beginning enrollment.

Importantly, while some of our findings were anticipated, such as concerns about infrastructural support and perceptions of imperfect transitional care processes, many findings were novel and unanticipated by the TNP team. For example, we did not anticipate interest in a transitional care program to work with non-VA hospitals, nor concerns about transportation for rural Veterans. We also did not foresee the extent to 
which clinicians we spoke to were pleasantly surprised by the time and effort expended in understanding processes, culture, and context at each expansion site. However, we could not address all potential barriers during implementation, such as transportation challenges and staffing issues at PACT clinics because these issues exceed the scope of the TN role. Understanding these potential barriers allowed us to help the TN's plan for these challenges.

Previous studies have used PRISM to assess barriers and facilitators prior to Implementation, ${ }^{18,19}$ but only in single-site programs. These studies found that participants felt that the intervention needed to be compatible with current processes and consider clinician time constraints along with unique patient needs. Our study corroborated this finding in a multisite pre-implementation evaluation. Using PRISM in a multisite implementation of a care coordination intervention provided the opportunity to identify barriers that are generalizable to implementation of care coordination interventions across sites with very different characteristics. We build on previous studies by identifying several facilitators and barriers related to five of six PRISM domains. Further, our study shows that PRISM can successfully integrate qualitative data from a variety of sources to identify actionable themes.

Many of the themes in our data may be applicable to other care coordination interventions within the VHA. For example, challenging communication infrastructures and difficulties meeting program demands are certainly not unique barriers to TNP. Our findings reveal challenges with critical steps to safe care transitions, including coordination with community and social support resources, coordinating care among team members, and outpatient follow-up. ${ }^{4}$ Knowing how to assess and effectively respond to such barriers is a critical step in the implementation process. The examples provided here were successful strategies for implementation and adaptation of an evidence-based care coordination intervention. Future studies should try to replicate these results to increase generalizability for care coordination interventions. We are currently implementing TNP at five additional sites and continue to streamline the use of PRISM for pre-implementation assessment.

\section{Limitations and Next Steps}

This evaluation was conducted by qualitative analysts, and methods may be difficult to replicate without similar resources to conduct site visits. We were unable to thoroughly assess patient perspective of the intervention due to a limited number of interviews with Veterans. We did not have the resources to collect data of comparable detail to that collected at VA hospitals and PACT clinics. Consideration of patient perspective is a key domain of PRISM, and future work should assess patient level barriers and facilitators related to care coordination programs. This study was conducted in the VA, and some of the contextual factors, facilitators, and barriers identified may be unique to the VA. Future studies should determine which contextual elements are present only in the VA, and which contextual elements influence the implementation of care coordination interventions in the community. Finally, there is no gold standard for validating our assessment. Further experience across future projects will help to refine our approach.

\section{CONCLUSIONS}

The use of an implementation science framework is crucial to multisite implementation of complex interventions. Using PRISM, we identified barriers and facilitators to TNP implementation, as well as contextual information that allowed us to adapt implementation to local settings. Results guided adaptations to program delivery and materials. Sites acted upon findings early in the implementation process. Common barriers and facilitators across TNP sites are likely generalizable to VHA care coordination interventions.

Acknowledgements: We would like to thank our operational partners from the VA Office of Rural Health and the VA Office of Nursing Services for their support of the TNP. We would like also to thank the many clinicians and staff who participated in interviews, observations, process mapping, and brainwriting activities.

Corresponding Author: Chelsea Leonard, PhD; Denver/Seattle Center of Innovation for Veteran-Centered and Value Driven Care VA Eastern Colorado Healthcare System, Denver, CO, USA (e-mail: Chelsea.leonard@va.gov).

Authors' Contributions $C L, H G, E L$, and RB jointly designed the study, conducted all analyses, and drafted the paper. MM, AM, LK, $B L$, and $A C$ assisted in data collection and manuscript revisions.

Funding Information This work was supported by the VA Office of Rural Health (visit www.ruralhealth.va.gov to learn more), which funded the Transitions Nurse Program (N19-FY14Q3-SO-P01240) in coordination with the VA Office of Nursing Services. The sponsor had no role in the design, conduct, analysis, interpretation, or presentation of the study. Dr. Burke was supported by a VA Career Development Award.

\section{Compliance with Ethical Standards:}

Conflict of Interest: The authors declare that they do not have a conflict of interest.

Disclaimer: The views expressed in this article are those of the authors and do not necessarily represent the position or policy of the Department of Veterans Affairs or the United States Government.

Ethics Approval: This study is a designated quality improvement by the VA Office of Rural Health. This project is not human subject research.

\section{REFERENCES}

1. Forster AJ, Murff HJ, Peterson JF, Gandhi TK, Bates DW. The incidence and severity of adverse events affecting patients after discharge from the hospital. Ann Intern Med. 2003;138(3):161-167. 
2. Auerbach AD, Kripalani S, Vasilevskis EE, Sehgal N, Lindenauer PK, Metlay JP, et al. Preventability and causes of readmissions in a national cohort of general medicine patients. JAMA Intern Med. 2016;176(4):484 493.

3. Burke RE, Kripalani S, Vasilevskis EE, Schnipper JL. Moving beyond readmission penalties: creating an ideal process to improve transitional care. J Hosp Med. 2013;8(2):102-109.

4. Burke RE, Guo R, Prochazka AV, Misky GJ. Identifying keys to success in reducing readmissions using the ideal transitions in care framework. BMC Health Serv Res. 2014;14(1):423.

5. Care Coordination [Internet]. Available at: https://www.ahrq.gov/professionals/prevention-chronic-care/improve/coordination/index.html. Accessed: 3 Aug 2018 .

6. Kilbourne AM, Hynes D, O'Toole T, Atkins D. A research agenda for care coordination for chronic conditions: aligning implementation, technology, and policy strategies. Transl Behav Med. 2018;8(3):515-521.

7. Williams MV, Li J, Hansen LO, Forth V, Budnitz T, Greenwald JL, et al. Project BOOST implementation: lessons learned. South Med J. 2014;107(7):455-465.

8. Naylor MD, Sochalski JA. Scaling up: bringing the transitional care model into the mainstream. Issue Brief Commonw Fund 2010;103(11):1-12.

9. Coleman EA, Berenson RA. Lost in transition: challenges and opportunities for improving the quality of transitional care. Ann Intern Med. 2004; 141(7):533-536.

10. Kind AJ, Brenny-Fitzpatrick M, Leahy-Gross K, Mirr J, Chapman E, Frey B, et al. Harnessing Protocolized Adaptation in Dissemination: Successful Implementation and Sustainment of the Veterans Affairs Coordinated-Transitional Care Program in a Non-Veterans Affairs Hospital. J Am Geriatr Soc. 2016;64(2):409-416.

11. Huynh AK, Hamilton AB, Farmer MM, Bean-Mayberry B, Stirman SW, Moin T, et al. A pragmatic approach to guide implementation evaluation research: strategy mapping for complex interventions. Front Public Health. 2018;6:134.

12. Joint Commission. Transitions of Care: The need for a more effective approach to continuing patient care. Available at: https://www. jointcommission.org/assets / 1/18/Hot_Topics_Transitions_of_Care.pdf. Accessed 23 July 2018.

13. Øvretveit $\mathbf{J}$. Understanding the conditions for improvement: research to discover which context influences affect improvement success. BMJ Qual Saf. 2011;20:i18-i23.

14. Squires JE, Graham ID, Hutchinson AM, Michie S, Francis JJ, Sales A, et al. Identifying the domains of context important to implementation science: a study protocol. Implement Sci. 2015;10(1): 135 .

15. Feldstein AC, Glasgow RE. A practical, robust implementation and sustainability model (PRISM) for integrating research findings into practice. Jt Comm J Qual Patient Saf. 2008;34(4):228-243.

16. Damschroder LJ, Aron DC, Keith RE, Kirsh SR, Alexander JA, Lowery JC. Fostering implementation of health services research findings into practice: a consolidated framework for advancing implementation science. Implement Sci. 2009;4(1):50.

17. Stetler CB, Damschroder LJ, Helfrich CD, Hagedorn HJ. A guide for applying a revised version of the PARIHS framework for implementation. Implement Sci. 2011;6(1):99.

18. Liles EG, Schneider JL, Feldstein AC, Mosen DM, Perrin N, Rosales AG, et al. Implementation challenges and successes of a populationbased colorectal cancer screening program: a qualitative study of stakeholder perspectives. Implement Sci. 2015;10(1):41.

19. Beck A, Bergman DA, Rahm AK, Dearing JW, Glasgow RE. Using implementation and dissemination concepts to spread 21st-century wellchild care at a health maintenance organization. Perm J. 2009;13(3):10.

20. Burke RE, Kelley L, Gunzburger E, Grunwald G, Gokhale M, Plomondon ME, et al. Improving transitions of care for veterans transferred to tertiary VA medical centers. Am J Med Qual. 2018;33(2):147-153.
21. Leonard C, Lawrence E, McCreight M, Lippmann B, Kelley L, Mayberry A, et al. Implementation and dissemination of a transition of care program for rural veterans: a controlled before and after study. Implement Sci. 2017;12(1): 123.

22. Saleem JJ, Plew WR, Speir RC, Herout J, Wilck NR, Ryan DM, et al. Understanding barriers and facilitators to the use of Clinical Information Systems for intensive care units and Anesthesia Record Keeping: A rapid ethnography. Int J Med Inform. 2015;84(7):500-11.

23. Gilmartin H, Lawrence E, Leonard C, McCreight M, Kelley L, Lippmann B, et al. Brainwriting premortem: a novel focus group method to engage stakeholders and identify pre-implementation barriers. J Nurs Care Qual. 34(2):94-100.

24. Jones J, Nowels CT, Sudore R, Ahluwalia S, Bekelman DB. The future as a series of transitions: qualitative study of heart failure patients and their informal caregivers. J Gen Intern Med. 2015;30(2):176-182.

25. Muhr T. ATLAS.ti. Berl Sci Softw Dev. GmbH. 1997.

Publisher's Note Springer Nature remains neutral with regard to jurisdictional claims in published maps and institutional affiliations.

\section{APPENDIX. KEY INFORMANT INTERVIEW GUIDE}

\section{Introduction}

Hello Ms./Mr./Dr. thank you for taking the time to speak with me today. I know how busy you are and I really appreciate your willingness to help us out with this interview. My name is I am a Qualitative Analyst with a team working to improve outcomes for rural Veterans after they are hospitalized at your facility. We would like to hear your thoughts on the current transition process for rural Veterans who return to their rural PACT after hospitalization at a tertiary VA hospital. We think that the TNP might help the transition process at your site, but we would appreciate your feedback on the proposed program. We are specifically interested in your insight as to how the Transitions Nurse might best function within your day-to-day context.

\section{Taping Procedure}

We would like to record this interview to ensure that we collect everything that is said, is that ok with you? That way, we can have an accurate record of what you say. If yes, we will ask you again when the audio recording begins. (Be sure to affirm.) In addition, we will take notes of what you say in case the tape-recorder malfunctions.

\section{Confidentiality}

Any comments you make here today will be confidential. Your name or any other identifying information will not be included in our report. We are interested in what you have to say, so we want you feel like you can speak freely.

Do you have any questions? [Refer to the TNP Description for Interviewees if there are questions] 
1. To start, can you tell me your title and role here at (name of facility)?

a. How long have you been in this role?

2. Could you please walk me though what happens when a rural Veteran is discharged, starting with the discharge decision, all the way to the first PCP follow-up?

a. What is your role in this process?

b. Can you tell me how...?

c. What do you mean by...?

d. Is this common transition?

e. Additional probes to help with process mapping, if participant is highly involved in discharge process (i.e. $R N$, discharge planner, social work, etc)

i. How does discharge process take place?

ii. When does the discharge planning begins?

iii. Who (what staff roles) is involved in getting the Veteran ready for discharge?

iv. Who specifically performs discharge patient counseling and medication reconciliation and at what point in the process?

v. Do you contact PACT teams about a discharged Veteran? How do you notify PACT that a Veteran is being discharged from VA Medical Center?

vi. Do you send any information to the PACT teams?

1. If yes, what kind of information and how do you send this information?

vii. Do you schedules follow up appointments for the Veteran as they are being discharged from your VA Medical Center?

3. What works well in the current process?

a. What doesn't work well in the current process?

b. Can you tell me how...?

c. What do you mean by...?

4. Can you describe your facility's relationship with rural PACT sites?

(PRISM: Characteristics of organizational recipients)

a. How is communication between your facility and rural PACT sites? i. Can you give me an example of....?

ii. Can you tell me about a time when....?
5. On a scale of $1-10,10$ being the most satisfied, how would you rate the patient-centeredness of the transition?

a. Can you tell me why you rated it X?

6. On a scale of $1-10,10$ being the most satisfied, how would you rate your satisfaction with the quality of the current process for transitioning rural Veterans back home/back to PACT?

a. Can you tell me more about why you rated it X?

7. On a scale of $1-10,10$ being the most efficient, how would you rate the current transitions process?

a. Can you tell me more about why you rated it $\mathrm{X}$ ?

Along with your calendar invite for this interview, we included a description of the TNP program, did you have a chance to take a look? If yes, continue. If no, please review the TNP one pager with the interview participant and ask them if they have any questions before proceeding.

8. Can you please describe your overall perception of the Rural Transitions Nurse Program for coordinating care for rural Veterans following an inpatient hospitalization?

(PRISM: Program (Intervention): Organizational perspective)

a. [Note] Be sure to assess positive aspects of TNP

i. What are or might be positive consequences of TNP?

ii. How confident are you that TNP will provide these positive consequences and why?

b. [Note] Be sure to assess concerns of TNP.

i. In your opinion, what are or might be negative consequences of TNP?

ii. In your opinion, what are or might be unintended consequences of TNP? 
9. What barriers, if any, do you think there would be to successfully implementing the TNP process?

(PRISM: Implementation and

Sustainability Infrastructure)

Probe on specific organizational context "signals" from administrative data

a. Can you give me an example of....?

b. What do you mean by...?

c. Do you have any suggestions to overcome these barriers?

(PRISM: Implementation and

Sustainability Infrastructure)

10. What do you think would facilitate/support the TNP process at your facility?

(PRISM: Implementation and Sustainability Infrastructure)

11. In your opinion, how will TNP affect patient care?

(PRISM: Program (Intervention): Organizational perspective)

a. What do you mean by...?

b. Can you give me an example of...?

12. How could TNP fit best in the process of coordinating care for rural Veterans?

(PRISM: Program (Intervention):

Organizational perspective)

a. Can you give me an example of....?

b. What do you mean by...?

c. What could be the most valuable service the transitions nurse can provide in the future?

13. How do you think the implementation of TNP will affect your job responsibilities/workload?

(PRISM: Characteristics of Organizational recipients)

a. Can you give me an example of....?

b. What do you mean by...?

c. How do you think it will affect the workload of other staff at your facility?
14. What types of incentives might encourage providers to support patients in the TNP process?

(PRISM: Implementation and

Sustainability Infrastructure)

a. Can you give me an example of....?

b. What do you mean by...?

15. Now I would like to ask you some final quick questions about the value of the Program.

a. What factors do you generally take into account when you evaluate the value of a service?

b. What kind of data or measures would you need to determine the value of the Transitions Nurse Program?

c. What results/findings would you want to see to continue supporting the Transitions Nurse Program?

16. Is there anything else you would like us to know regarding the Rural Transitions Nurse Program?

Follow up

Would it be ok if we contact you with any follow up questions after we review your responses? Do you have any questions before we begin? 